Draft Version November 4, 2018

Preprint typeset using $\mathrm{IAT}_{\mathrm{E}} \mathrm{X}$ style emulateapj v. 08/13/06

\title{
THE RADIO JET INTERACTION IN NGC 5929: DIRECT DETECTION OF SHOCKED GAS
}

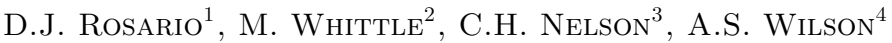 \\ Draft version November 4, 2018
}

\begin{abstract}
We report the discovery of kinematic shock signatures associated with a localized radio jet interaction in the merging Seyfert galaxy NGC 5929. We explore the velocity-dependent ionization structure of the gas and find that low ionization gas at the interaction site is significantly more disturbed than high ionization gas, which we attribute to a local enhancement of shock ionization due to the influence of the jet. The characteristic width of the broad low-ionization emission is consistent with shock velocities predicted from the ionization conditions of the gas. We interpret the relative prominence of shocks to the high density of gas in nuclear environment of the galaxy and place some constraints of their importance as feedback mechanisms in Seyferts.

Subject headings: galaxies: Seyfert - galaxies: jets - line: profiles — shock waves — galaxies: interactions
\end{abstract}

\section{INTRODUCTION}

Most Seyfert AGN are associated with weak nuclear radio sources which show radio spectral indices and morphologies (if resolved) consistent with synchrotronemitting jets and lobes (Ulvestad \& Wilson 1984; Nagar et al. 1999), though their physical properties are poorly constrained. However, they are effective avenues of kinetic and thermal feedback from the active nucleus and may play an important role in determining the evolution of the central spheroid.

In the small fraction of Seyferts with kpc-scale radio jets, several studies have uncovered clear signatures of interactions between the jet and surrounding gas, in the form of disturbed emission line profiles in the inner Narrow-Line Region (NLR), as well as close associations between resolved jet structures and NLR gas (e.g. Whittle et al. 1988; Capetti et al. 1996; Falcke et al. 1998; Cooke et al. 2000; Cecil et al. 2002; Whittle \& Wilson 2004). Depending on the physical make-up of the jet, relativistic or ram pressure can drive fast shocks, compressing, sweeping up and altering the appearance of the NLR. Postshock gas, with a temperature of several $10^{7}$ $\mathrm{K}$, is a source of ionizing photons which couple the shock properties to the ionization state of the surrounding gas (e.g. Dopita \& Sutherland 1996). Shocks and winds can also change the distribution of ISM phases in the NLR by destroying and ablating clouds (Fragile et al. 2005). While the active nucleus is usually the dominant source of ionization even in strongly jetted Seyferts (e.g. Whittle et al. 2005), widespread shocks driven by a jet can alter the ionization of the NLR by affecting the properties of the ISM.

Studies of shock structure and energetics (Dopita \& Sutherland 1996; Allen et al. 2008) predict strong differences between the emission line spectrum of dense post-

\footnotetext{
${ }^{1}$ Astronomy Department, University of California, Santa Cruz, CA 95064; rosario@ucolick.org

2 Astronomy Department, University of Virginia, Charlottesville, VA 22903; dmw8f@virginia.edu

${ }^{3}$ Physics and Astronomy, Drake University, Des Moines, IA 50311-4505; charles.nelson@drake.edu

4 Astronomy Department, University of Maryland, College Park, MD 20742; deceased
}

shock gas and gas that is ionized by, but not in direct dynamical contact with, the shock (the precursor). Therefore, a clear signature of shock ionized gas is a difference between the line profiles of low and high ionization lines, which are preferentially produced by post-shock and precursor gas, respectively (Whittle et al. 2005).

In this work, we present an HST/STIS spectroscopic study of NGC 5929, a local Seyfert galaxy with a wellstudied bi-polar radio jet. Previous ground-based spectroscopic studies find evidence of a localized interaction between the jet and the near-nuclear emission line gas (Whittle et al. 1986; Wakamatsu \& Nishida 1988; Taylor et al. 1989; Wilson \& Keel 1989; Ferruit et al. 1997). The datasets and analysis methods used are briefly reviewed in $\S 2$. Direct shock features and a picture of the interaction are developed in $\S 3$ and $\S 4$. We discuss the role of shocks in Seyferts and AGN feedback in $§ 5$. NGC 5929 has a systemic heliocentric velocity of $c z=2492 \mathrm{~km}$ $\mathrm{s}^{-1}$ based on the stellar aborption line redshift of Nelson \& Whittle (1995), which corresponds to $161 \mathrm{pc} \operatorname{arcsec}^{-1}$ $\left(\mathrm{H}_{0}=75 \mathrm{~km} \mathrm{~s}^{-1} \mathrm{Mpc}^{-1}\right)$.

\section{OBSERVATIONS AND REDUCTIONS}

\subsection{STIS Dataset}

Our principal dataset is two medium-dispersion STIS G430M long-slit spectra covering the lines of the [O III] $\lambda \lambda 5007,4959$ doublet and $\mathrm{H} \beta$ (HST Program: GO 8253; PI : Whittle). The data was spectrophotometrically calibrated using the CALSTIS pipeline. For each spatial increment, the continuum was modeled using a lower order polynomial and subtracted. These spectra were combined to generate a continuum-free $2-\mathrm{D}$ spectrum from both STIS slits The equivalent width of $\mathrm{H} \beta$ is always greater than $10 \AA$ in the NLR of NGC 5929, and $\approx 140 \AA$ at the location of the interaction. Therefore, corrections to the emission line strengths and profiles due to Balmer absorption are negligible.

\subsection{HST and Radio Images}

Emission line maps of NGC 5929 in [OIII] $\lambda \lambda 4959,5007+\mathrm{H} \beta$ were prepared from archival HST narrow/medium-band WF/PC-1 images (Program: GO 3724; PI: Wilson). Details of these maps can be 


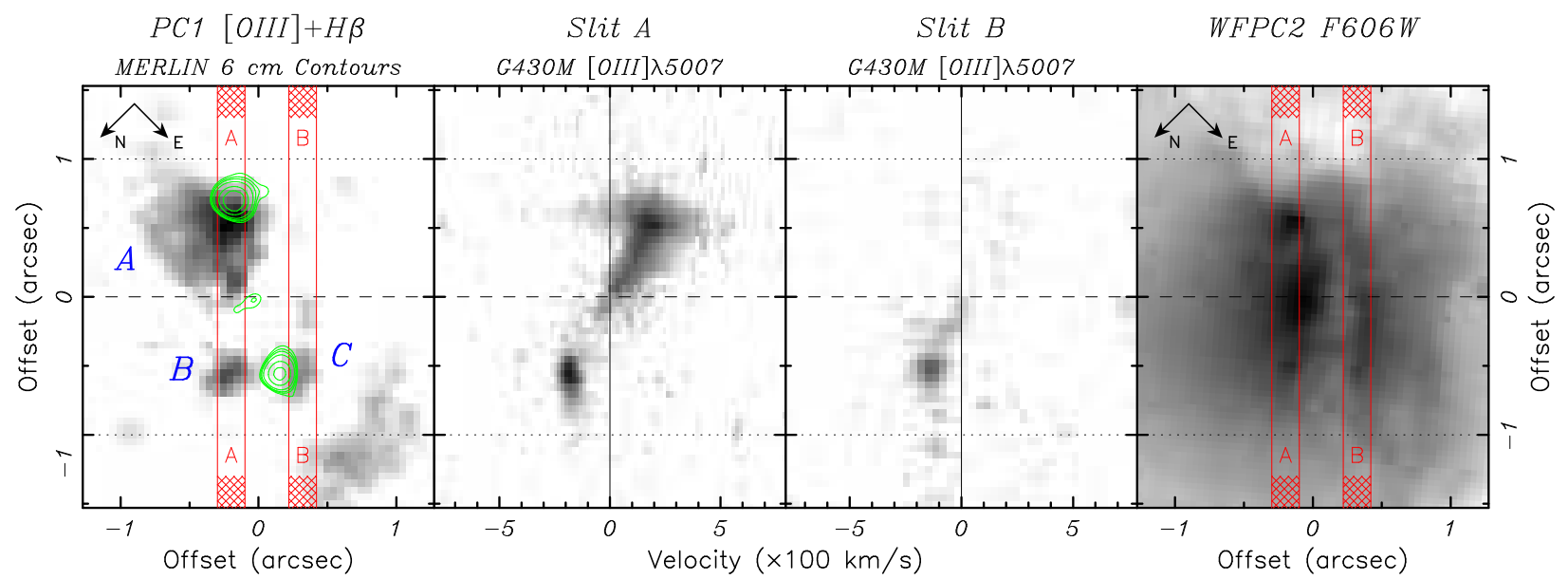

FIG. 1. - Panels of images and spectra of the nuclear region of NGC 5929. The 2D spectra in Panels 2 and 3 correspond to STIS long-slit apertures A and B respectively, as indicated on the images plotted in Panels 1 and 4 . The radio map (gaussian smoothed by 0 .' 3 to bring out its structure) is plotted in Panel 1 in contours.

TABLE 1

STIS OBSERVATIONS ${ }^{\dagger}$

\begin{tabular}{cccc}
\hline \hline Aperture & Dataset & Exp. (s) & Nuc. Offset (") \\
\hline NGC 5929 A & O5G403010 & 1524 & 0.198 \\
NGC 5929 B & O5G403020 & 600 & 0.390
\end{tabular}

${ }^{\dagger}$ Date: 07/02/00; Grating: G430M; $\Delta \lambda\left(\AA \mathrm{pix}^{-1}\right): 0.277 ; \mathrm{PA}:-134^{\circ} 6$

found in Bower et al. (1994). In addition, a high S/N F606W WFPC2 archival image of the galaxy was used to trace the stellar and dust geometry in and around the NLR. A reduced and calibrated $5 \mathrm{GHz}$ radio map of NGC 5929 was obtained from the MERLIN archive (http://www.merlin.ac.uk/archive/). This data was first presented in Su et al. (1996).

The various HST datasets were registered to a common astrometric frame using image cross-correlation techniques. The dust lane in NGC 5929 crosses the NLR, effectively obscuring the true position of the nucleus. Lattanzi et al. (1997) use the astrometric information of stars in the WF/PC-1 images of the galaxy to compare the locations of the continuum peak and the unresolved core in a MERLIN radio map. Assuming the radio core corresponds to the true nucleus, they find that the $\mathrm{WF} / \mathrm{PC}-1$ continuum peak is offset by $0^{\prime \prime} 1 \pm 0.05 \mathrm{NW}$ from the radio core. We have included this small correction in our final registered images (see Panel 4 in Figure 1 for a sense of the amount of correction involved).

\section{THE NLR OF NGC 5929}

\subsection{Descriptive Framework}

NGC 5929 is part of a major galaxy merger with NGC 5930 (Arp 90). Clear signs of tidal tails and disturbed gaseous kinematics are seen in ground-based two-dimensional spectra of the merger system (Lewis \& Bowen 1993), and twisting, filamentary dust structure is visible in the nuclear region (Panel 4 of Fig. 1)

The radio source has been imaged at high resolution with the VLA (Ulvestad \& Wilson 1984; Wilson \& Keel 1989) and MERLIN (Su et al. 1996). It exhibits a triple structure, with two bright compact steep-spectrum hotspots on either side of a faint flat-spectrum core that is unresolved even in the highest resolution maps. Low surface brightness emission joins the hotspots to the radio core along $\mathrm{PA} 61^{\circ}$, which is also the rough orientation of the elongated emission line distribution.

The diverse imaging and spectroscopic datasets are showcased together in Figure 1. In Panel 1, the $[\mathrm{O}$ III $]+\mathrm{H} \beta$ emission line image is displayed in greyscale, with the STIS slit positions and radio map contours overplotted. Three emission line regions, identified by Bower et al. (1994) are labelled A, B and C. The SW radio hotspot coincides with the edge of Region A, while the NW radio hotspot lies near Region B. From the broadband image in Panel 4, the emission line features are revealed as parts of a possibly contiguous region, crossed by a dust lane that obscures the line and continuum emission and modulates the appearance of the NLR. This dust structure is part of a filamentary network extending to several kpc (Malkan et al. 1998).

The $[\mathrm{OIII}] \lambda 5007$ line from the two medium-resolution STIS spectra are plotted in Panels $2 \& 3$ of Figure 1. The slits are oriented NE-SW from the bottom to the top of the panel, and a dashed horizontal line marks the reference position on the slit, i.e, the point along the slit closest to the nucleus of the galaxy. Solid vertical lines indicate the systemic velocity.

The [O III] line from both STIS spectra shows clear velocity structure. A detailed treatment of the kinematics of the NLR is not the aim of this Letter and will be addressed in a later paper. A brief description will suffice here. Slit A traverses the brighter emission line regions and therefore gives the best picture of the ionized gas kinematics. The velocity of the line peak increases along this slit from almost systemic at the reference position to a maximum value of $+185 \mathrm{~km} \mathrm{~s}^{-1}$ at a nuclear radius of $\sim 0$ ". $65 \mathrm{SW}(104 \mathrm{pc})$. This broad gradient is mirrored, though less clearly, to the NE in both Slits A and $\mathrm{B}$. The gradient between the nucleus and Regions B and $\mathrm{C}$ are similar in both slits, implying that both Regions are part of the same gaseous complex, bisected in projection by the dust lane. The FWHM of the [O III] line goes through a rapid transition from $\sim 125 \mathrm{~km} \mathrm{~s}^{-1}$ to greater than $200 \mathrm{~km} \mathrm{~s}^{-1}$ at a nuclear distance of 0.5 in the brightest portion of Region A. Regions B and C, on the other hand, exhibit uniformly narrow line profiles.

\subsection{Ionization Conditions}




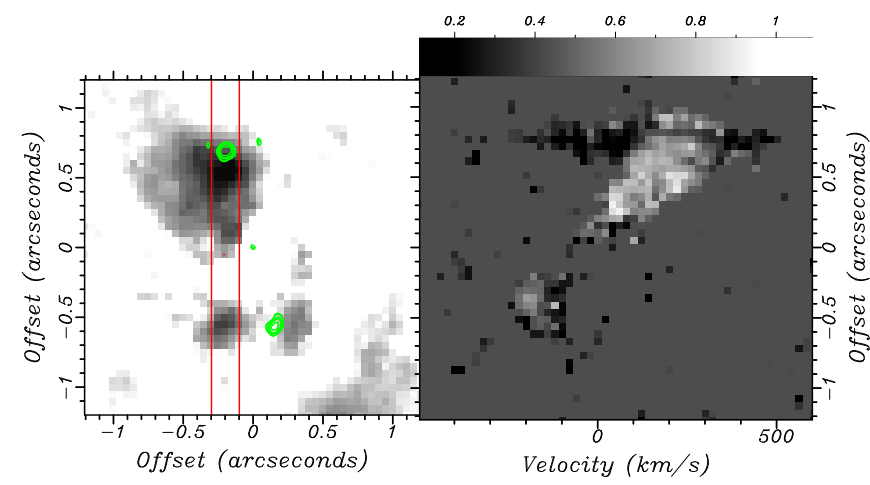

Fig. 2.- The [OIII] image with MERLIN $6 \mathrm{~cm}$ contours overlayed (left) and the $[\mathrm{OIII}] / \mathrm{H} \beta$ log ratio map (right). The location of the slit aperture is indicated on the image as a rectangle, corresponding to the spatial range of the ratio map. A grayscale lookup table is plotted in the bar above the map. A fiducial value of 0.43 is used to mask regions with no significant line emission, which helps to improve the visibility of the ratio map.

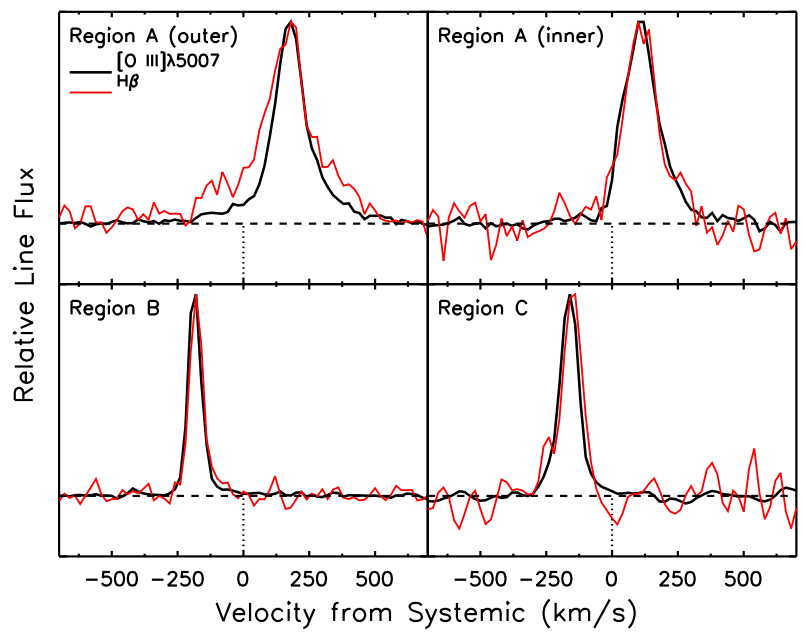

FIG. 3.- A comparison of the line profiles of the [O III] line (black) and $\mathrm{H} \beta$ line (red) in four parts of the NGC 5929 NLR. The lines have been scaled to a common peak value. The jet interaction, in the outer part of Region $\mathrm{A}$, is associated with broad $\mathrm{H} \beta$, unlike any other part of the emission line region.

In Figure 2, a map of the $[\mathrm{OIII}] \lambda 5007 / \mathrm{H} \beta$ line ratio from the Slit A spectrum is plotted, alongside the WF/PC-1 [O III] image, overlayed with the contours of the $5 \mathrm{GHz}$ radio map and the boundaries of the STIS aperture. The ratio image was created by first rebinning the two-dimension spectrum of each line onto a common velocity range and then dividing them to generate a map of the $[\mathrm{O} \mathrm{III}] / \mathrm{H} \beta$ ratio as a function of velocity and slit position. This ratio of is quite sensitive to ionization state, yet insensitive to dust reddening.

The $[\mathrm{O}$ III $] \lambda 5007 / \mathrm{H} \beta$ ratio is in the range of $2-6$, intermediate between Seyferts and LINERs (Kewley et al. 2006). Variations in ionization are seen across the NLR. The average excitation of Region $\mathrm{B}$ is towards the low end of the observed range, with average $[\mathrm{O}$ III $] \lambda 5007 / \mathrm{H} \beta \sim 3$, while the inner part of Region $\mathrm{A}$ is more highly ionized ([O III $] \lambda 5007 / \mathrm{H} \beta \sim 5)$. However, surrounding the position of the bright knot in Region A, a sharp drop in the ratio is seen. Interestingly, this change in ionization is velocity dependent. In the high velocity wings of the lines, the $[\mathrm{O} \mathrm{III}] / \mathrm{H} \beta$ drops to almost unity implying a very low ionization state, while the central core of the lines remain at a more modest ionization. This trend is different from most Seyferts where the high velocity gas tends to be of equal or higher ionization than low velocity material (e.g., Pelat et al. 1981).

This difference between the average ionization of the NLR and Region A is brought out well by comparing the velocity profiles of $[\mathrm{O}$ III $] \lambda 5007$ and $\mathrm{H} \beta$ in different parts of the NLR, as is done in Figure 3. The width of the $\mathrm{H} \beta$ line compared to [O III] is substantially higher in Region A, compared to both the inner part of the same Region and the profiles in Region B and C.

What is the reason for this peculiar ionization behaviour? We believe this results from strong shocks driven by the radio plasma into the ISM. The compressed gas in the post-shock cooling zone is expected to share the high velocities of the shock, yet is predicted to have $[\mathrm{O}$ III $] / \mathrm{H} \beta$ in the range of $0.7-4.0$, depending on the shock speed and local magnetic field (Dopita \& Sutherland 1996). The enhancement of line emission from shocks and possibly a precursor region, over and above the emission from gas photoionized by the AGN, could then account for the appearance of the bright emissionline knot in Region A associated with the radio hotspot. A moderate contribution to the line emission from shocks is also consistent with the HST spectroscopic study of Ferruit et al. (1999). From the relative strengths of the [SII] $\lambda \lambda 4069,4077$ and $\lambda \lambda 6717,6731$ lines, which are produced profusely in the post-shock cooling zone, they estimate high temperatures (greater than 20,000-50, $000 \mathrm{~K}$ ) and somewhat low electron densities (around $300 \mathrm{~cm}^{-3}$ ) for the $[\mathrm{SII}]$-emitting gas. These values are quite reasonable for normal diffuse ISM that has been compressed by a shock front. Based on a simple double gaussian decomposition of the $\mathrm{H} \beta$ line integrated across the knot, we estimate an approximate linewidth of $420 \mathrm{~km} \mathrm{~s}^{-1}$ for the broad component, which includes the high velocity wings. This nicely matches the predicted shock velocities from Ferruit et al. (1999) which were based on comparisons to shock ionization models of Dopita \& Sutherland (1996). While it is difficult to directly associate shock speeds with integrated line kinematics, this broad consistency adds credence to a shock-driven origin for the emission of the knot in Region A.

\section{THE NATURE OF THE JET INTERACTION}

Our combination of high spatial resolution optical spectroscopy and imaging provide the best existing view of the compact jet-ISM interaction in NGC 5929. High velocity gas is only seen at the location of the southwestern radio hotspot, while in other parts of the NLR, linewidths are narrow and a velocity gradient expected from virial motion is seen, consistent with ground-based slit and IFU spectroscopy (Keel 1985; Stoklasová et al. 2009).

We adopt a simple model for the jet interaction, following that of Whittle et al. (1986). The radio jet plows through the ISM as it propagates outward, driving strong shocks at its head (the radio hotspot). As this shocked gas cools and gets denser, it becomes the [S II]-emitting gas described by Ferruit et al. (1999) and creates the broad $\mathrm{H} \beta$ emission. Allen et al. (2008) model the prop- 
erties of post-shock gas as a function of shock velocity $V_{s h}$, pre-shock density and magnetic field strength. Taking a magnetized shock as fiducial, the [S II]-derived densities and $V_{s h} \sim 400 \mathrm{~km} \mathrm{~s}^{-1}$ (from the linewidth of the broad $\mathrm{H} \beta$ component) imply a pre-shock density of $n \sim 30 \mathrm{~cm}^{-3}$ and a shock cooling length around tens of parsecs. The pre-shock density estimate has a large uncertainty and could be as low as $1 \mathrm{~cm}^{-3}$ if the shocks are weakly magentized, which would also decrease the cooling length significantly. However, since Seyfert jets are generally associated with $\mathrm{mG}$ level magnetic field, we adopt our estimates for magnetic shocks as most likely. The cooling length we derive is resolved in our HST images and may explain the small offset of 0 .' 15 between the location of the radio hotspot and the peak of the line emission in Region A. This interpretation is very tentative, since patchy dust obscuration is the region may also cause such an offset.

$\mathrm{Su}$ et al. (1996) estimate flux densities, radio spectral indices and source sizes for the various components of the radio source, from which we calculate radio source equipartition pressures, using the relations of $\mathrm{Mi}$ ley (1980) and assuming that the radio emitting plasma has a filling factor of unity and an ion fraction $a=10$, and that the radio spectrum extends from $0.01 \mathrm{GHz}$ to $100 \mathrm{GHz}$ with a constant spectral index of -0.82 . The equipartition synchrotron pressure of the radio hotspot is $10^{-7}$ dyne $\mathrm{cm}^{-2}$, which may be compared to the ram pressure of the shock front, $m_{p} n V_{s h}^{2} \sim 8 \times 10^{-8}$ dyne $\mathrm{cm}^{-2}$. The two pressures are comparable, consistent with the view that the shocked gas surrounds and confines the radio hotspot. It is worthwhile to note that such pressures are considerably higher than those typically found in Seyfert radio sources, which may indicate that this is a relatively young jet ejection event. Eventually, interactions with the surrounding gas will confine the jet flow into a static lobe, like those found in late-stage interactions (Capetti et al. 1996; Whittle \& Wilson 2004).

\section{DISCUSSION: SHOCK SIGNATURES IN SEYFERTS}

Why do we see such obvious signatures of shocks in this object and not in others? This may be due to the weakness of the AGN: NGC 5929 has an absorptioncorrected hard X-ray luminosity of $1.8 \times 10^{41} \mathrm{erg} \mathrm{s}^{-1}$ - low compared to average local Seyferts (Cardamone et al. 2007). Or perhaps the nuclear environment of the galaxy is dense and gas-rich due to its ongoing merger. This will lower the ionization parameter of nuclear radia- tion and produce a more compact emission line region. In both scenarios, the influence of the nucleus at larger radii will be relatively unimportant, making shock ionized line emission visible against the general background of centrally ionized gas. If this is indeed the case, it implies that radiative shock ionization from nuclear outflows is widespread in Seyfert NLRs, but is usually secondary to nuclear photoionization processes and only visible in low-luminosity Seyferts or those with dense nuclear environments. A rich nuclear environment raises another possibility that the jet is impacting a dense molecular cloud, enhancing the shock luminosity. This may explain why no clear shock signatures are seen around the NE radio hotspot, though the obscuration of the main dust lane prevents a direct view of this region.

The luminosity of the broad $\mathrm{H} \beta$ component is $8 \times 10^{38}$ erg s${ }^{-1}$. Following Dopita \& Sutherland (1996), the $\mathrm{H} \beta$ flux scales with the total radiative flux from the shock, with a weak dependence on $V_{s h}$, giving shock luminosities of $5 \times 10^{41} \mathrm{erg} \mathrm{s}^{-1}$ - comparable or slightly less than the total luminous output of the AGN (taking a X-ray bolometric correction of $\sim 10$ ). Using standard relationships from Osterbrock (1989), the $\mathrm{H} \beta$ luminosity can be used to derive the mass of ionized gas in the broad component: $3 \times 10^{4} \mathrm{M}_{\odot}$. If this mass of gas was accelerated to $V_{s h}$, it would have a total kinetic energy of $5 \times 10^{52}$ ergs. Taking the approximate acceleration timescale to be the crossing time of the region of size $0 . ! 3\left(48 \mathrm{pc}\right.$ ) at $V_{s h}$ (around $10^{5}$ $\mathrm{yr}$ ), a lower limit on the 'kinetic luminosity' of the jet is estimated to be $1.5 \times 10^{40} \mathrm{erg} \mathrm{s}^{-1}$. This is few to several percent of the total AGN energy output. Given that jet outflows are a relatively common feature of Seyfert activity and couple strongly to the ISM through shocks, jet driven feedback can effectively carry as much as energy as the AGN photon luminosity to kpc scales, and transfer a tenth or less of this energy in the form of kinetic energy to the NLR. This can have important consequences for the suppression of bulge star-formation and the energy budget and dynamics of circum-nuclear gas.

We thank the referee for their helpful review. DR acknowledges the support of NSF grants AST-0507483 and AST-0808133. Based on observations made with the NASA/ESA Hubble Space Telescope. MERLIN is a National Facility operated by the University of Manchester at Jodrell Bank on behalf of STFC.

\section{REFERENCES}

Allen, M.G, et al. 2008, ApJS, 178, 20

Baldwin, J.A., Wilson, A.S., \& Whittle, M. 1987, ApJ, 319, 84

Bower, G.A. et al. 1994, AJ, 107, 1686

Capetti, A., Axon, D.J., Macchetto, F., Sparks, W.B., \& Boksenberg, A. 1996, ApJ, 469, 554

Cardamone, C.N, Moran, E. C, \& Kay, L.E 2007, AJ, 134, 1263

Cecil, G., et al. 2002, ApJ, 568, 627

Cooke, A.J., et al. 2000, ApJS, 129, 517

de Vaucouleurs, G., de Vaucouleurs, A., Corwin, H.G., Buta, R.J.,

Paturel, G., and Fouqué, 1991, "Third Reference Catalogue of Bright Galaxies", Springer-Verlag

Dopita, M.A, \& Sutherland, R.S. 1996, ApJS, 102, 161

Falcke, H., Wilson, A.S., \& Simpson, C. 1998, ApJ, 502, 1999

Ferruit, P., Pécontal, E., Wilson, A.S., \& Binette, L. 1997, å, 328, 493
Ferruit, P., Wilson, A.S., Whittle, M., Simpson, C., Mulchaey, J.S., \& Ferland, G.J. 1999, ApJ, 523, 147

Fragile, C.P., Anninos, P., Gustafson, K., \& Murray, S.D. 2005, ApJ, 619, 327

Hao, L., et al. 2005, AJ, 129, 1795

Heckman, T.M., Miley, G.K., van Breugel, W.J.M., \& Butcher, H.R. 1981, ApJ, 247, 403

Keel, W.C. 1985, Nature, 318, 43.

Kewley, L.J., Groves, B., Kauffmann, G., \& Heckman, T. 2006, MNRAS, 372, 961

Lattanzi, M.G., Capetti, A., \& Macchetto, F.D. 1997, A\&A, 318, 997

Lewis, J.R., \& Bowen, D.V. 1993, MNRAS, 264, 818

Malkan, M.A., Gorjian, V., \& Tam, R. 1998, ApJS, 117, 25

Miley, G.H. 1980, ARA\&A, 18, 165 
Nagar, N., Wilson, A.S., Mulchaey, J.S., \& Gallimore, J.F. 1999, ApJS, 120, 209

Nelson., C.H., \& Whittle, M. 1995, ApJS, 99, 67

Osterbrock, D.E. 1989, Astrophysics of Gaseous Nebulae and Active Galactic Nuclei (University Science Books)

Pelat, D., Alloin, D, \& Fosbury, R.A.E. 1981, MNRAS, 195, 787,

Su, B.M., Muxlow, T.W.B., Pedlar, A., Holloway, A.J., Steffen, W., Kukula, M.J., \& Mutel, R.L. 1996, MNRAS, 279, 1111

Stoklasová, I., Ferruit, P., Emsellem, E., Jungwiert, B., Pécontal, E., \& Sánchez, S. 2009, A\&A, 500, 1287

Taylor, D., Dyson, J.E., Axon, D.J., \& Pedlar, A. 1989, MNRAS, 240, 487

Ulvestad, J.S., \& Wilson, A.S. 1984, ApJ, 285, 439
Ulvestad, J.S., \& Wilson, A.S. 1989, ApJ, 343, 659

Wakamatsu, K., \& Nishida, M.T. 1988, ApJ, 325, 596

Whittle, M. et al., 1988, ApJ, 326, 125

Whittle. M., Haniff, C.A. Ward, M.J., Meurs, E.J.A, Pedlar, A.,

Unger, S.W., Axon, D.J., \& Harrison, B.A. 1986, MNRAS, 222, 189

Whittle, M., \& Wilson, A.S. 2004, AJ, 127, 606

Whittle, M., Rosario, D.J., Silverman, J.D., Nelson, C.H., \& WIlson, A.S., 2005, AJ, 129, 104

Wilson, A.S., \& Keel, W.C 1989, AJ, 98, 5 